\title{
Screening and Identification of Chitinolytic Actinomycetes and Study on the Inhibitory Activity against Turfgrass Root Rot Disease Fungi
}

\author{
Qi Wang1, Bihua Duan2*, Rui Yang², Yaorong Zhao1, Lu Zhang1 \\ ${ }^{1}$ College of Landscape Architecture, Beijing University of Agriculture, Beijing, China \\ ${ }^{2}$ College of Plant Science and Technology, Beijing University of Agriculture/Beijing Key Laboratory for \\ Agricultural Application and New Technique, Beijing, China \\ Email: dbhbac@sohu.com, leibiyijue@126.com
}

Received December 2014

\begin{abstract}
Objective: This study aimed to separate and pure chitinase producing actinomycetes with stronger activity from lawn soils of Beijing. Method: Morphology and culture characteristics were observed. physiological and biochemical characteristics were detected. 16SrDNA sequence was analyzed. Result: The highest chitinase activity strain was Bn035, whose relative diameter of transparent circle $\mathrm{K} 2$ was 3.42 at which the chitinase activity of fermentation broth reached a maximum value of $1.23 \mathrm{U} / \mathrm{mL}$ in the 6th day. Crude enzyme solution and bacteria cake of strain Bn035 generated stronger bacterial resistance to all root rot fungi which indicated that the strain had a strong broad-spectrum resistance to root rot fungi. Conclusion: The strain was identified as Streptomyces scabrisporus. It will be a potential biological control function.
\end{abstract}

\section{Keywords}

Strain Bn035, Transparent Circle, Chitinase Activity, Antagonism, 16SrDNA

\section{Introduction}

Chitin is the main component of fungal cell wall, Chitin $\left(\mathrm{C}_{8} \mathrm{H}_{13} \mathrm{O}_{5} \mathrm{~N}\right) \mathrm{n}$ is straight chain polymer with $\beta$-1,4-Nacetyl glucosamine as the basic unit. Chitinase can be found throughout the natural world (such as in microbial, plant, animal), Chitinase from microorganisms is well studied and thoroughly researched [1]. Chitinase can degrade fungi chitin to glucosamine monomers, dimers and trimers, thereby undermining fungal cell walls, and further inhibiting the growth of fungal hyphae, thus achieving biocontrol effects [2]. According to reports, chitinase crude extract from Bacillus circulans has different inhibitory effect on five species of fungi (Verticillium dahliae, Trichoderma vivide, Rrizoctonia solani, Fusarium oxysporum, Gaeumannomyces graminis) [3]. Man-

${ }^{*}$ Corresponding author.

How to cite this paper: Wang, Q., Duan, B.H., Yang, R., Zhao, Y.R. and Zhang, L. (2015) Screening and Identification of Chitinolytic Actinomycetes and Study on the Inhibitory Activity against Turfgrass Root Rot Disease Fungi. Journal of Biosciences and Medicines, 3, 56-65. http://dx.doi.org/10.4236/jbm.2015.33009 
dana Zarei [4] report that chitinase from Serratia marcescens B4A have strong inhibitory effects on four species of fungi (Rhizoctonia solani, Bipolaris sp., Alternaria raphani, Alternaria brassicicola).

Turfgrass root rot disease is caused by various pathogens through individual or combined infection, the lesions occurred mainly in the roots and rhizomes, in the early stage of the disease, the stems and leaves turn yellow, then wilt in the late stage of disease, thus reducing the landscape value of lawn. Fusarium sp., Rhizoctonia sp., Pythium sp., Biploaris sp. are the main pathogens of turfgrass root rot disease, at the same time, Drechslera sp., and Curuularia sp. can cause symptoms of turfgrass root rot diseases [5] [6]. Lawns are increasingly becoming important landscape in people's lives, but there are relatively few research reports on the prevention and control of turfgrass root rot pathogens, and research usually focus on traditional chemical control, the shortcoming of chemical control is that it is time-consuming and laborious, the effect is of short duration, and can cause environmental pollution [7] [8]. Therefore, it is important to research on prevention and treatment of turfgrass root rot and focus on finding new method of prevention and control, in this study, we explore the biological control measures, based on the principle of chitin degradation by chitinase, starting from lawn soil samples, we isolated several strains of actinomycetes that efficiently produce chitinase, we isolate the individual strain of actinomycetes and identify the inhibitory effect of its chitinase on turfgrass root rot pathogens, our research has practical application in the biological control of turfgrass root rot disease.

\section{Materials and Methods}

\subsection{Collection and Processing of Soil Samples}

A total of 225 soil samples were collected from 45 locations of artificial turf and natural grass in nine urban areas (Beijing, Shijiazhuang, Chengde, Hohhot, Weinan, Taiyuan, Datong, Jinan, Zhengzhou). The method of soil processing is as described by Feng Yinan, Yang Runqing [9].

\subsection{Preparation of Culture Medium, Inhibitors and Colloidal Chitin}

Gao 1th synthetic medium, PDA culture medium, physiological and biochemical medium, observations culture medium are prepared as described by Zhao Juan [10], chitin liquid medium and chitin agar medium are prepared as described by Kolla [11].

Chitin is prepared as described by Gao Xiaoning [12], after preparation, chitin is heat sterilized and stored in refrigerator at $4^{\circ} \mathrm{C}$.

To inhibit the growth of bacteria and fungi, $10 \%$ potassium dichromate solution is used as described by Yang Haiying [13].

\subsection{The Pathogenic Fungi for Experimental Test}

Bipolaris sorokiniana (No. ACCC36529), Fusarium oxysporum (No. ZB4), Rhizoctonia solani (No. ZB3), Pythium capsici (No. ZB7) are the turfgrass root rot fungi in our experiment, and are kindly provided by the research group of Li Shaojie in Chinese Academy of Microbiology Research.

\subsection{Screening of Actinomycetes Stains That Produce Chitinase}

We use the dilution plate method. Soil suspension was diluted to the concentration of $10^{-3} \mathrm{~g} \cdot \mathrm{mL}^{-1}$, then take 0.2 $\mathrm{mL}$ of soil suspension, sterile coating on CA flat plate, invert the plate and culture at $28^{\circ} \mathrm{C}$ in constant temperature incubator for 14 days, observe the colony formation and chitin-lytic zone. The colonies with relatively large chitin-lytic zone were picked for purification culture, the chitin-lytic zone and colony diameter was measured using colony method; the fungus cake method is used for rescreening, punch holes (diameter $=4 \mathrm{~mm}$ ) in the areas of culture medium with good and dense colony growth, pick the fungus cake, invert it in the CA flat plate, for each CA flat plate, we put in three fungus cakes, and culture at $28^{\circ} \mathrm{C}$ in constant temperature incubator for 14 days, the chitin-lytic zone and colony diameter was measured using fungus cake method, the colonies with relatively large chitin-lytic zone were selected and stored in refrigerator at $4^{\circ} \mathrm{C}$.

\subsection{Determination of Actinomyces Chitinase Activity}

Using DNS (3,5-dinitrosalicylic acid ) method of reducing sugars [14] for the determination of chitinase activity. 
From $30^{\circ} \mathrm{C}$ shake flask fermentation, take $10 \mathrm{~mL}$ and centrifuge it at the speed of $5000 \mathrm{r} \cdot \mathrm{min}^{-1}$ for $10 \mathrm{~min}$ at $4^{\circ} \mathrm{C}$, collect the supernatant, prepare four centrifuge tube $(1.5 \mathrm{~mL})$, add $0.6 \mathrm{~mL}$ supernatant into each centrifuge tube, one of them is the control tube, directly inactivated in a $100^{\circ} \mathrm{C}$ water bath for $10 \mathrm{~min}$, afterwards $0.7 \mathrm{~mL}$ colloidal chitin (1\% mass fraction, $\mathrm{pH}$ 6.0) is added into the control tube and then placed in $45^{\circ} \mathrm{C}$ water bath; the other three tubes are for duplication experimental tests, $0.7 \mathrm{~mL}$ colloidal chitin (1\% mass fraction, $\mathrm{pH} 6.0)$ were added into each tube, and then placed in $45^{\circ} \mathrm{C}$ water bath for $60 \mathrm{~min}$, and subsequently inactivated in a $100^{\circ} \mathrm{C}$ water bath for $10 \mathrm{~min}$, after cooling, the tubes are centrifuged at the speed of $15,000 \mathrm{r} \cdot \mathrm{min}^{-1}$ for $10 \mathrm{~min}$, from each tube, collect $1 \mathrm{~mL}$ supernatant (distilled water is used as blank control) and put it into a 25 mL tube already containing $1.5 \mathrm{~mL}$ DNS, then add $1 \mathrm{~mL}$ of distilled water, and place the tubes in a $100^{\circ} \mathrm{C}$ water bath for $6 \mathrm{~min}$, then put the tubes into running water to cool, afterwards adding distilled water to set the volume of each tube at $4 \mathrm{~mL}$, shake the tube and then let it settle for $20 \mathrm{~min}$, followed by OD540 measurement.

The definition of enzyme activity unit (U): under the condition of $40^{\circ} \mathrm{C}$ and pH6.0, the amount of hydrolysis enzyme required to produce the equivalent of $1 \mathrm{ug} \mathrm{N}$-acetylglucosamine per minute from hydrocolloid chitin.

\subsection{Determination of Actinomyces Inhibitory Effects on Pathogenic Fungi}

We use the liquid culture method [12]. The fungus cake (diameter $=4 \mathrm{~mm}$ ) containing each of the four pathogenic fungi is treated with $0.2 \mathrm{~mL}$ crude enzyme solution in the culture medium of $20 \mathrm{~mL}$ potato dextrose (PD), each treatment group was repeated three times, $0.2 \mathrm{~mL}$ sterile water instead of $0.2 \mathrm{~mL}$ crude enzyme solution is used in the control group, shaking culture $\left(30^{\circ} \mathrm{C}, 180 \mathrm{r} \cdot \mathrm{min}^{-1}\right)$ for 2 days, then filtering and drying the pathogens hyphae, record the dry weight of pathogens hyphae, and calculate the inhibitory rate, inhibitory rate $=($ the dry weight of pathogens hyphae in the control group - the dry weight of pathogens hyphae in the treatment group) $\times$ (the dry weight of pathogens hyphae in the control group $\times 100 \%)^{-1}$.

We use the flat confrontation method [15]. The fungus cake containing experimental actinomyces were inoculated symmetrically at $25 \mathrm{~mm}$ around the center of PDA medium plate, each treatment group was repeated three times, after incubation at $28^{\circ} \mathrm{C}$ constant temperature for 5 days, the fungus cake of pathogenic fungi was inoculated in the center of the culture plate, then continue incubation at $28^{\circ} \mathrm{C}$ constant temperature, until the pathogens hyphae growth reach plate edge in the control side without actinomyces inoculation (the incubation time is 4 days for Bipolaris sorokiniana, 5 days for Fusarium oxysporum, 4 days for Rhizoctonia solani, 2 days for Pythium capsici), then measure the diameter of inhibition, and calculate the inhibitory rate, inhibitory rate $=$ (pathogenic fungi diameter in the control side - pathogenic fungi diameter in the confrontation side) $\times(\mathrm{patho-}$ genic fungi diameter in the control side $\times 100 \%)^{-1}$.

\subsection{Identification of Actinomyces}

1) The culture characteristics of actinomycetes: According to the method described in the International Streptomyces Plan (ISP), we cultured actinomycetes with standard medium and common medium at $28^{\circ} \mathrm{C}$ for 14 days, then observed the culture characteristics of mycelium growth and color, as well as the soluble pigment.

2) The morphological characteristics of actinomycetes: We cultured actinomycetes with Gao 1th synthetic medium at $28^{\circ} \mathrm{C}$ for a period between 7 days and 14 days, then using the insert method and scanning electron microscope respectively, to observe the morphological characteristics of base mycelium, aerial hyphae, spore wire and spore body [16].

3) The chemical component of actinomycetes cell wall: Using the method of rapid thin layer chromatography [17], we analysis the whole-cell amino acids, as well as the sugar composition of whole-cell hydrolysates.

4) The physiological and biochemical characteristics of actinomycetes: To analysis the physiological and biochemical characteristics of actinomycetes, we conducted the following experiments: carbon utilization, starch hydrolysis, cellulose decomposition, gelatin liquefaction test, milk coagulation and peptonize, hydrogen sulfide production, melanin production, nitrate reduction [18].

5) The 16S rRNA sequence analysis: The genomic DNA extraction from actinomycetes are based on the method of Xu Ping [19], using genomic DNA as template, with universal primers 27F and 1492R (27F:

5'-AGAGTTTGATCCTGGCTCAG-3', 1492R: 5'-TACGGYTACCTTGTTACGACTT-3') in a $25 \mu \mathrm{L}$ system $\left(10 \times\right.$ Buffer (Mg-) $2.5 \mu \mathrm{L}, \mathrm{Mg}^{2+} 1.5 \mu \mathrm{L}$, dNTP (2.5 mM) $1.0 \mu \mathrm{L}$, Primer 16S-27-F 1.0 $\mu \mathrm{L}$, Primer 16S-1492-R $1.0 \mu \mathrm{L}$, Template DNA $0.5 \mu \mathrm{L}$, Taq DNA Polymerase ( $\left.2 \mathrm{U} \cdot \mu \mathrm{L}^{-1}\right) 0.3 \mu \mathrm{L}$, dd $\mathrm{ddH}_{2} \mathrm{O}$ to $25.0 \mu \mathrm{L}$ ), we conducted PCR amplification (first denaturation at $94^{\circ} \mathrm{C}$ for $4 \mathrm{~min}$; followed by 30 cycles of denaturation at $94^{\circ} \mathrm{C}$ for $1 \mathrm{~min}$, 
annealing at $56^{\circ} \mathrm{C}$ for $1 \mathrm{~min}$, extension at $72^{\circ} \mathrm{C}$ for $2 \mathrm{~min}$; final extension at $72^{\circ} \mathrm{C}$ for $7 \mathrm{~min}$ ). PCR products are purified using gel extraction kit, DNA sequencing conducted by Beijing Liuhe Genomics Technology Corporation. We analysis the sequences through Blast Homology Search in the GenBank database, representative strains were selected for similarity analysis using CLUSTALX2.0 software, and phylogenetic tree was constructed using the Neighbor-Joining method of Mega4.0 software.

\section{Results and Analysis}

\subsection{The Screening of Chitinase-Producing Actinomycetes Strains}

From the total of 225 soil samples, we isolated 63 strains of actinomycetes that can form chitin-lytic zone on chitin plate, we picked 5 strains of actinomycetes with the largest ratio of chitin-lytic zone to colony diameter, after purification and further rescreening with fungus cake method, we measure and calculate the ratio of chitin-lytic zone to colony diameter, the results indicate that Bn035 strain has strong capacity to degrade chitin, its ratio of chitin-lytic zone to colony diameter is 3.60 and 3.42 in the initial screening and rescreening, respectively (Table 1), therefore Bn035 strain was selected for further study, using the fungus cake method, it can form clear and obvious chitin-lytic zone (Figure 1).

\subsection{The Effect of Incubation Time on Chitinase Activity of Bn035 Strain}

Bn035 strain was incubated in chitin liquid medium from 1 day to 8 days, we take samples of the fementation medium at each day of incubation, the samples were centrifuged and the supernatant analyzed for chitinase activity, the results showed that chitinase activity of Bn035 strain increased significantly on the third day of incubation, and reached the maximum value of $1.23 \mathrm{U} \cdot \mathrm{mL}^{-1}$ on the sixth day of incubation (Table 2).

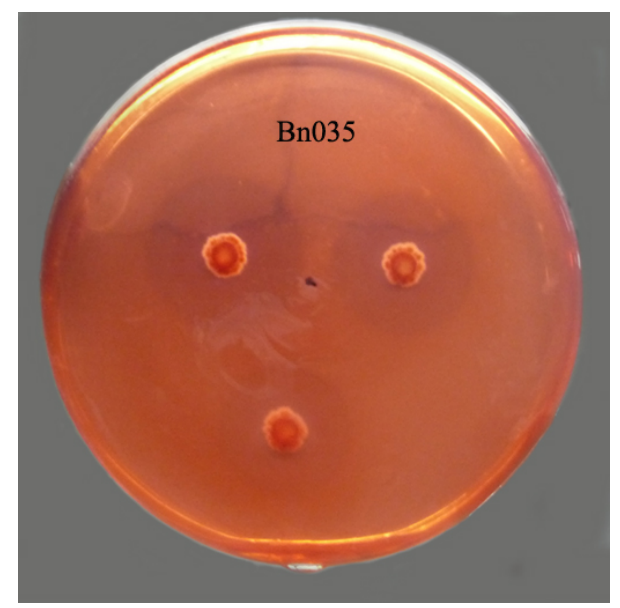

Figure 1. Lytic zone was produced by bacteria cakes of Bn035 in chitin agar medium.

Table 1. The chitin-lytic zone test results from 5 strains producing chitinase in the process of screening and rescreening.

\begin{tabular}{|c|c|c|c|c|c|c|}
\hline Strains No. & $\begin{array}{c}\text { Diameter of } \\
\text { chitin-lytic zone } \\
\text { from colonies/mm }\end{array}$ & $\begin{array}{c}\text { Diameter from } \\
\text { colonies/mm }\end{array}$ & $\begin{array}{l}\text { Relative diameter } \\
\text { of chitin-lytic zone } \\
\text { from colonies }\end{array}$ & $\begin{array}{c}\text { Diameter of } \\
\text { chitin-lytic zone } \\
\text { from bacteria cakes/mm }\end{array}$ & $\begin{array}{l}\text { Diameter from } \\
\text { bacteria } \\
\text { cakes } / \mathrm{mm}\end{array}$ & $\begin{array}{l}\text { Relative diameter } \\
\text { of chitin-lytic zone } \\
\text { from bacteria cakes }\end{array}$ \\
\hline $\mathrm{Bn} 023$ & $20.17 \pm 0.21^{\mathrm{a}}$ & $8.03 \pm 0.15^{\mathrm{a}}$ & $2.51 \pm 0.02^{\mathrm{d}}$ & $13.23 \pm 0.15^{\mathrm{e}}$ & $10.13 \pm 0.12^{\mathrm{d}}$ & $1.31 \pm 0.01^{\mathrm{c}}$ \\
\hline $\mathrm{Bn} 024$ & $14.23 \pm 0.15^{\mathrm{e}}$ & $6.10 \pm 0.10^{\mathrm{b}}$ & $2.33 \pm 0.01^{\mathrm{e}}$ & $20.17 \pm 0.15^{\mathrm{b}}$ & $18.03 \pm 0.06^{\mathrm{a}}$ & $1.12 \pm 0.02^{\mathrm{e}}$ \\
\hline Bn026 & $17.30 \pm 0.26^{\mathrm{c}}$ & $6.13 \pm 0.12^{\mathrm{b}}$ & $2.82 \pm 0.01^{\mathrm{c}}$ & $19.27 \pm 0.21^{\mathrm{c}}$ & $16.13 \pm 0.06^{\mathrm{b}}$ & $1.19 \pm 0.01^{\mathrm{d}}$ \\
\hline $\mathrm{Bn} 027$ & $15.03 \pm 0.21^{\mathrm{d}}$ & $5.00 \pm 0.10^{c}$ & $3.01 \pm 0.02^{\mathrm{b}}$ & $15.30 \pm 0.26^{\mathrm{d}}$ & 11.1. $\pm 010^{c}$ & $1.38 \pm 0.01^{\mathrm{b}}$ \\
\hline Bn035 & $18.60 \pm 030^{b}$ & $5.17 \pm 0.06^{\mathrm{c}}$ & $3.60 \pm 0.03^{a}$ & $24.30 \pm 0.26^{\mathrm{a}}$ & $7.10 \pm 0.10^{\mathrm{e}}$ & $3.42 \pm 0.02^{\mathrm{a}}$ \\
\hline
\end{tabular}

Note: Values in the table are mean $\pm \mathrm{SD}, \mathrm{n}=3$. Different small letters in the same column indicates significant difference $(\mathrm{P}<0.05)$. 
Table 2. Chitinase activity of Bn035 after different incubation time.

\begin{tabular}{cccc}
\hline Incubation time/d & Chitinase activity/U·mL ${ }^{-1}$ & Incubation time/d & Chitinase activity/U.mL $^{-1}$ \\
\hline 1 & $0.08 \pm 0.04^{\mathrm{f}}$ & 5 & $1.01 \pm 0.01^{\mathrm{c}}$ \\
2 & $0.22 \pm 0.03^{\mathrm{e}}$ & 6 & $1.23 \pm 0.03^{\mathrm{a}}$ \\
3 & $0.75 \pm 0.02^{\mathrm{d}}$ & 7 & $1.12 \pm 0.04^{\mathrm{b}}$ \\
4 & $0.79 \pm 0.02^{\mathrm{d}}$ & 8 & $1.09 \pm 0.03^{\mathrm{b}}$ \\
\hline
\end{tabular}

Note: Values in the table of chitinase activity are mean \pm SD. $n=3$. Different small letters in the same column indicates ignificant difference $(\mathrm{P}<$ $0.05)$.

\subsection{The Inhibitory Effect of Bn035 against 4 Turfgrass Root Rot Fungi}

Using two methods (liquid culture method and plate confrontation method) respectively, we tested the inhibitory effect of Bn035 against 4 turfgrass root rot fungi, the two methods all indicate that the inhibitory effect of Bn035 against 3 turfgrass root rot fungi (Bipolaris sorokiniana, Fusarium oxysporum, Rhizoctonia solani) are very strong, the inhibitory effect of Bn035 against Pythium capsici is relatively strong; in addition, compared with plate confrontation method, liquid culture method shows better inhibitory results (with the exception of Fusarium oxysporum) (Figure 2, Table 3).

\subsection{Identification of Bn035 Strain}

\subsubsection{Morphological Characteristics and Culture Characteristics}

Bn035 is Gram-positive strain, its base mycelium has no cross and no broken, its aerial hyphae grow into cluster with leaf-like morphology and horizontal branching, its spore wire has straight or spiral morphology, its spores body has the shape of oval or cylindrical and wrinkle surface (Figures 3-5). With coating and inoculation on 12 kinds of culture medium, Bn035 spores were cultured at $28^{\circ} \mathrm{C}$ for a period of $7-10$ days, then observe the culture characteristics, Bn035 can grow generally well on the culture medium, the common color of mycelium is yellow or white, we have not observed any soluble pigment on the 12 kinds of culture medium.

\subsubsection{Analysis of Cell Wall Type, Physiological and Biochemical Characteristics of Actinomycetes} Bn035 cell walls contain the amino acids of L, L-DAP and glycine, the type of cell wall is type I; the whole-cell hydrolysates do not contain special sugar, the sugar type belongs to $C$ type (Figure 6). The main physiological and biochemical characteristics of Bn035 strain: it can hydrolysis starch and cellulose, it can reduce nitrate, it has weak positive reaction in milk coagulation and peptonize test, it cannot produce hydrogen sulfide and melanin, the test result of gelatin liquefaction is negative; the test result of carbon utilization is that Bn035 can use a lot of carbon sources, including D-glucose, sucrose, D-galactose, D-xylose, L-arabinose, L-rhamnose, D-mannitol, L-inositol, maltose, cellobiose, glycerol, D-raffinose, lactose, mannitol; Bn035 can use a lot of nitrogen sources, including peptone, yeast extract, $\left(\mathrm{NH}_{4}\right)_{2} \mathrm{SO}_{4}, \mathrm{NH}_{4} \mathrm{NO}_{3}$, L-asparagine.

\subsubsection{S rRNA Sequence Analysis}

After PCR amplification, Bn035 16S rRNA is sequenced, the gene fragment is 1406 bp in length (Genbank Accession No. KC440853), through BLAST analysis of related sequences in NCBI database, 11 species of Streptomyces with high homology were selected to construct phylogenetic tree, in which Bn035 strain and Streptomyces scabrisporus (EU841545.1) cluster in the same branch, with a homology of 99.9\%, therefore Bn035 strain is preliminarily identified as Streptomyces scabrisporus (Figure 7).

\section{Discussion}

\subsection{Screening Methods for Chitin-Degrading Capacity of Actinomycetes}

In the screening of chitinase-producing actinomycetes stains, based on the previously established colony method, we designed a new method, namely the fungus cake method, in this study, both methods are used in the comprehensive screening of target strains. In the initial screening, we use the colony method to select 5 strains with relatively large diameter of chitin-lytic zone, after continuous culture for five generations, the selected strains 


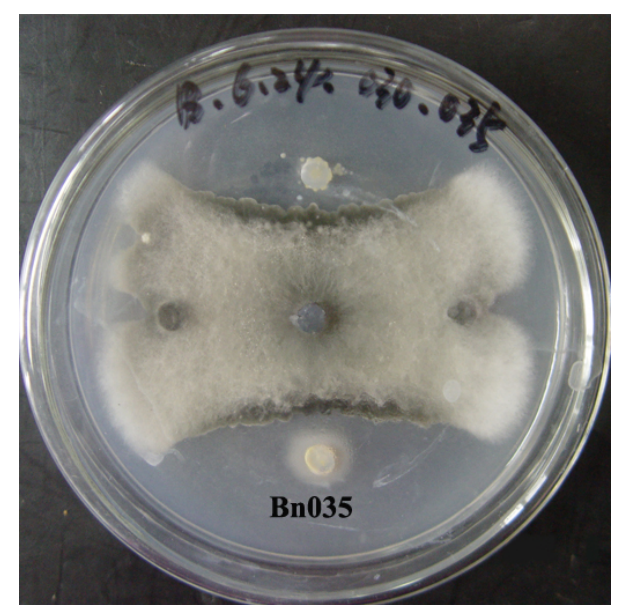

Figure 2. Inhibition of Bn035 against Bipolaris sorokiniana.

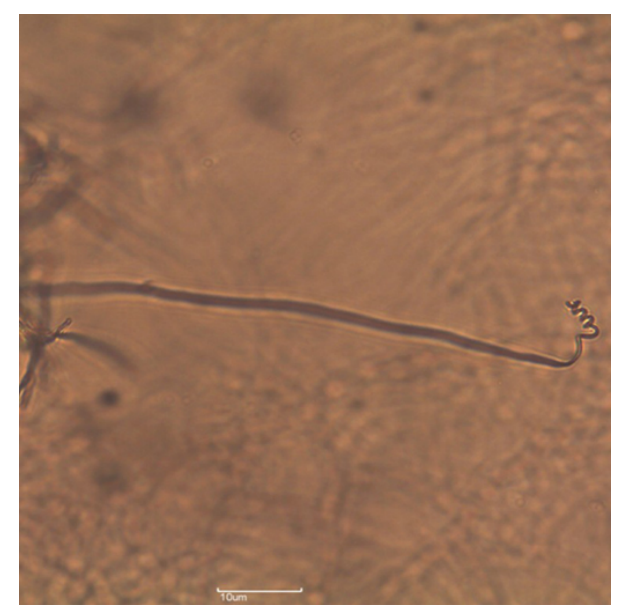

Figure 3. Aerial hyphae, spores wire of Bn035 spores/OM 400×.

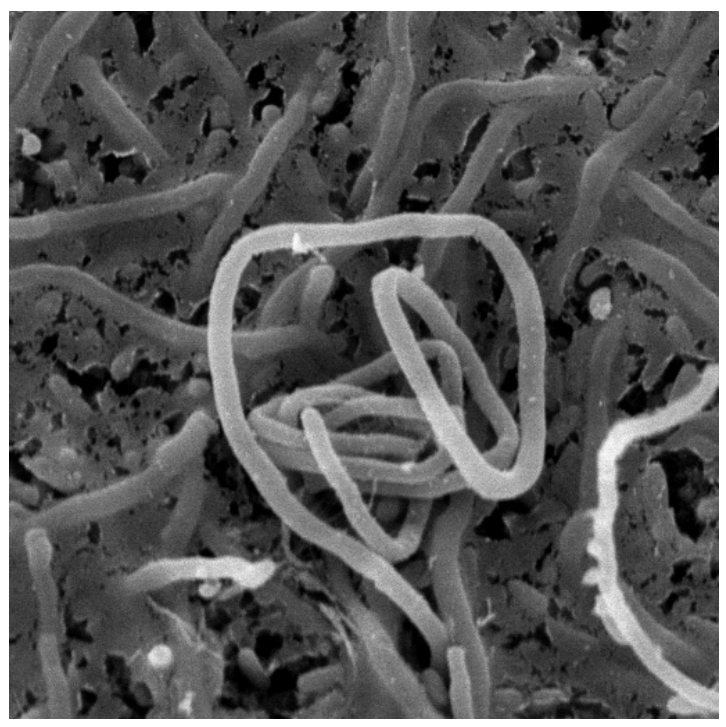

Figure 4. Spores wire of Bn035/SEM10950×. 


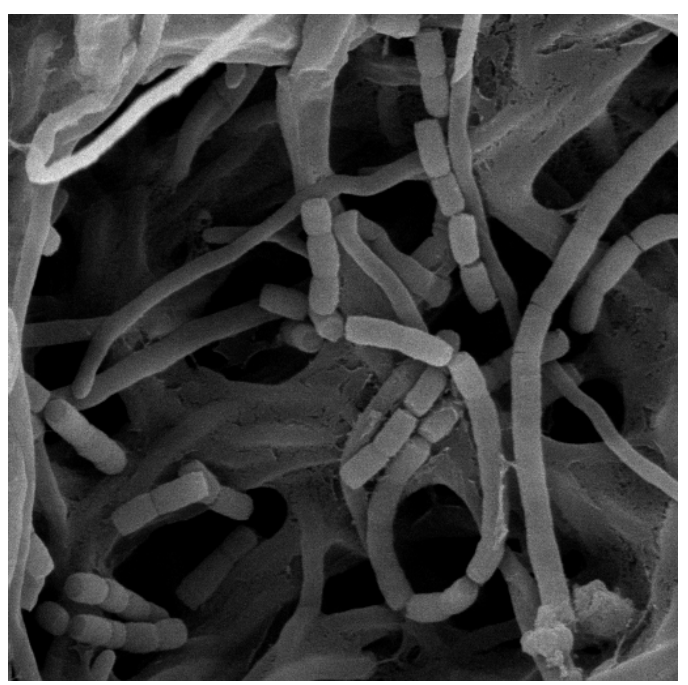

Figure 5. Spores of Bn035/SEM13000×.

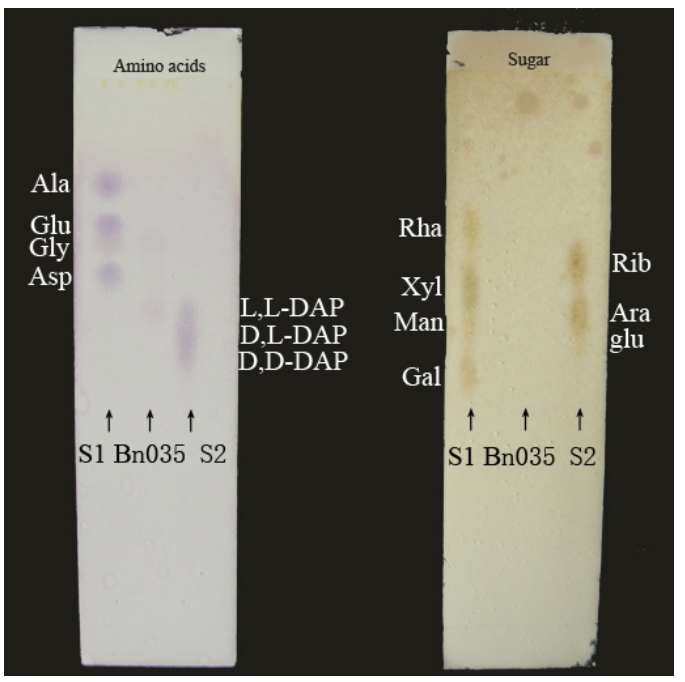

Figure 6. Thin layer chromatography results of Bn035.

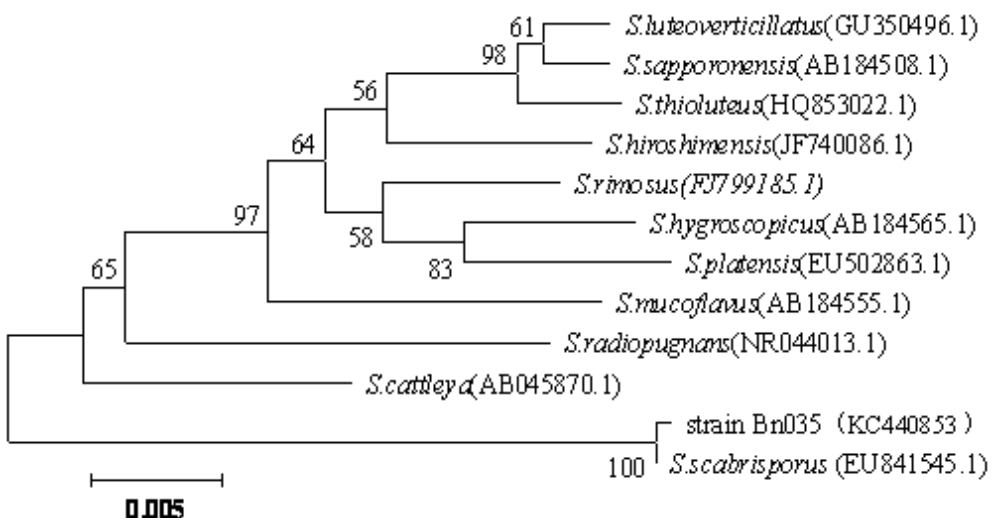

Figure 7. Phylogenetic tree of actinomycete Bn035 and other related Streptomyces strains based on $16 \mathrm{~S}$ rDNA. Note: GenBank accession number is in parentheses, support percentage of bootstrap (1000 samples) is at each branch point, the scale represents $0.5 \%$ sequence divergence. 
Table 3. The inhibition results of Bn035 against 4 turfgrass root rot fungi.

\begin{tabular}{ccc}
\hline Fungi of indication & Inhibition rate from flat confrontation method/\% & Inhibition rate from liquid culture method/\% \\
\hline Bipolaris sorokiniana & $57.03 \pm 3.20^{\mathrm{a}}$ & $74.90 \pm 3.36^{\mathrm{b}}$ \\
Fusarium oxysporum & $54.70 \pm 2.00^{\mathrm{a}}$ & $35.13 \pm 1.86^{\mathrm{c}}$ \\
Rhizoctonia solani & $42.00 \pm 2.81^{\mathrm{b}}$ & $84.63 \pm 2.19^{\mathrm{a}}$ \\
Phytophthora capsici & $20.00 \pm 2.52^{\mathrm{c}}$ & $26.80 \pm 2.42^{\mathrm{d}}$ \\
\hline
\end{tabular}

Note: Values in the table are mean $\pm \mathrm{SD}, \mathrm{n}=3$. Different small letters in the same column indicates significant difference $(\mathrm{P}<0.05)$.

are rescreened using the fungus cake method, the rescreening results show that, after five generations of continuous culture, the chitin-degrading capacity of Bn035 strain remains stable and strong, whereas the chitindegrading capacity of the other 4 strains decreased significantly, this difference perhaps due to strain degeneration after continuous culture [20], which also demonstrate that Bn035 strain can maintain efficient chitindegrading capacity after continuous culture, this characteristics provide a good foundation for the construction of genetic engineered strain. Furthermore, as shown in the relative diameter of the 5 strains obtained from both methods (the colony method and the fungus cake method), the deviation of relative diameters are quite small, indicating that the data obtain from both methods are very reliable, therefore we can use either the colony method or the fungus cake method for screening, but the fungus cake method as designed in our experiment is simple and easy to measure and photograph.

\subsection{Chitinase Activity and Inhibitory Effect}

There is a correlation between the chitinase activity of actinomycete and its inhibitory effect, but some strains with strong chitinase activity has no obvious inhibitory effect [21]. Zarei and others [4] studied the microscopic structure of chitin after being degraded by chitinase, their conclusion is that due to differences in the chemical structure of fungal chitin, therefore the inhibitory effect of chitinase against different kinds of pathogenic fungi vary significantly.

Through screening test, we isolated the Bn035 strain, on the 6th day of shake flask fermentation in CYS culture medium, its enzyme activity reached a maximum of $1.23 \mathrm{U} \cdot \mathrm{mL}^{-1}$, which is similar to the enzyme activity of Streptomyces longisporoflavus (GKSHJA) as reported by Gao Xiaoning [12], but the crude enzyme solution of Streptomyces longisporoflavus (isolated by Gao Xiaoning) have inhibitory rate of $48.3 \%$ and $7.9 \%$ against Rhizoctonia solani and Fusarium oxysporum, respectively, whereas the crude enzyme solution of Bn035 strain (obtained by us) have inhibitory rate of $84.63 \%$ and $35.13 \%$ against Rhizoctonia solani and Fusarium oxysporum, respectively. As suggested by Zarei [4], the crude enzyme solution of Bn035 strain have different inhibitory rate against the 4 kinds of pathogenic fungi, the chitinase produced by Bn035 strain have strong inhibitory effect against Rhizoctonia solani and Bipolaris sorokiniana, therefore Bn035 strain is potentially valuable in the prevention and biology control of related fungal disease. Using the plate confrontation method, Bn035 strain demonstrate strong inhibitory effect against 3 turfgrass root rot fungi (Bipolaris sorokiniana, Fusarium oxysporum, Rhizoctonia solani), but the liquid culture method showed better inhibitory results (with the exception of Fusarium oxysporum), the reason maybe that the crude enzyme solution can generally attach better to fungal hyphae, rapidly degrade the chitin, thereby efficiently inhibit fungal growth and reproduction [4], but in the case of Fusarium oxysporum, compared with the plate confrontation method, the liquid culture method showed lower inhibitory rate, the reason maybe that through plate confrontation method, Bn035 strain can produce other antimicrobial substances or effects, thereby increasing the inhibitory rate [22]. Furthermore, because the pathogenic fungal hyphae have strong growth and dispersal ability, its nutrition competition capability is far better than that of actinomycetes, especially in the case of Pythium capsici, when cultured at $28^{\circ} \mathrm{C}$ for 1 day, it can rapidly grow and spread to the entire plate, therefore in the experimental test of inhibitory effect, different kinds of pathogenic fungi should be treated differently.

\subsection{Research of the Isolated Strains and Their Application Prospect}

In this study, we isolated the Bn035 strain, identified as Streptomyces scabrisporus, this strain has not yet been thoroughly studied both in China and around the world, and most of the literature only described the basic cha- 
racteristics of this strain, such as this strains can produces antibiotics Hitachimycin [23], our study found that Bn035 strain can produce chitinase with relatively strong enzyme activity, and Bn035 strain has different degree of inhibitory effect against 4 representative pathogenic fungi that cause turfgrass root rot disease, Bn035 strain can effectively inhibit the pathogenic fungi, therefore this function has potential application in biological control, we need to further study the practical application of Bn035 strain. On the basis of field experiments that fully confirmed the field control effect of this strain, future research can be conducted in the optimization of fermentation conditions for enzyme production, as well as the cloning of chitinase gene from this strain.

\section{Conclusion}

In this study, through screening from soil samples of the lawn and meadow, we isolated Bn035 stain that can produce chitinase with strong enzyme activity. Its chitinase activity has the following characteristics: the relative diameter of chitin-lytic zone was 3.60 and 3.42 respectively; the enzyme activity reached a maximum of 1.23 $\mathrm{U} \cdot \mathrm{mL}^{-1}$ on the sixth day of shake flask fermentation. Crude enzyme solution and thallus of Bn035 strain showed strong antifungal activity against 4 representative pathogenic fungi that cause turfgrass root rot disease. The Bn035 stain was identified as Streptomyces scabrisporus by its morphological, cultural, physiological and biochemical characteristics, and 16S rRNA sequence analysis.

\section{Support}

Supported by the National Natural Foundation of China Project (31000551) (3097012) and the Natural Foundation of Beijing Project (5152006).

\section{References}

[1] Xiong, J. (2009) Isolation and Identification of a Chitinase-Producing Strain and Characteration of Its Chitinase. Central South University, Changsha, 13.

[2] Velusamy, P. and Kim, K.Y. (2011) Chitinolytic Activity of Enterobacter sp. KB3 Antagonistic to Rhizoctonia solani and Its Role in the Degradation of Living Fungal Hyphae. International Research Journal of Microbiology, 2, 206-214.

[3] Tan, J.-X., Chen, Z.-Y., Zhang, J., et al. (2001) Isolation and Identification of Chitinolytic Strains and Preliminary Study on the Antifungal Activity of Its Crude Chitinases. Plant Protection, 27, 1-3.

[4] Zarei, M., Aminzadeh, S., Zolgharnein, H., et al. (2011) Characterization of a Chitinase with Antifungal Activity from a Native Serratla marcescens B4A. Brazilian Journal of Microbiology, 42, 1017-1029. http://dx.doi.org/10.1590/S1517-83822011000300022

[5] Shi, J., Li, J.-C., Liu, Y.-Y., et al. (2000) A Primary Study on Pathogen of Turfgrass Root Rot Disease. Acta Agriculturae Boreali-Sinica, 15, 94-98.

[6] Sun, B.-J., Yuan, H.-X., Xing, X.-P., et al. (2007) Identification of Turfgrass Root Rot Disease in Zhengzhou. Grassland and Turf (Bimonthly), 125, 51-54.

[7] Zhang, P.-Z., He, T. and Wu, Y. (2004) Progress of Research on Fungicide Triadimefon in Agriculture of China. Journal of Zhejiang University of Science and Technology, 16, 28-34.

[8] Ji, S.-Q., Duan, Q.-F., Xu, G.-C., et al. (2006) Counter Measures against Preventing and Controlling Hyphal Cluster Root-Rot Sickness of White Leaf Lawn. Journal of Pingdingshan Institute of Technology, 15, 48-49.

[9] Feng, Y.-N. and Yang, R.-Q. (2010) Progress of Research on Measures of Isolation and Identification in Actinomycete Strains. Biotechnology, 20, 95-98.

[10] Zhao, J., Xue, Q.-H., Wang, L.-N., et al. (2011) Antagonistic Effect of Multifunctional Actinomycete Strain Act12 on Soil-Borne Pathogenic Fungi and Its Identification. Chinese Journal of Eco-Agriculture, 19, 394-398. http://dx.doi.org/10.3724/SP.J.1011.2011.00394

[11] Narayana, K. and Vijayalakshm, M. (2009) Chitinase Production by Streptomyces sp. ANU 6277. Brazilian Journal of Microbiology, 40, 725-733. http://dx.doi.org/10.1590/S1517-83822009000400002

[12] Gao, X.-N., Gulpiye, Wang, M.-Y., et al. (2010) Screening of Plant Endophytic Actinomycetes Producing Chitinase and Its Antagonistic Activity against Sclerotinia sclerotiorum. Journal of Zhejiang University (Agric1 \& Life Sci1), 36, 615-622.

[13] Yang, H.-Y., Du, G. and Lu, K.-F. (2008) Effect of Bacteriostatic on the Isolation of Actinomycetes. Hubei Agricultural Sciences, 47, 435-437.

[14] Zhao, K., Xue, P.-J. and Gu, G.-Y. (2008) Study on Determination of Reducing Sugar Content Using 3,5-Dinitrosali- 
cylic Acid Method. Food Science, 29, 534-536.

[15] Fang, Z.-D. (2007) Research Methods on Plant Disease. China Agriculture Press, Beijing, 246-249.

[16] Ruan, J.-S. and Huang, Y. (2011) Rapid Identification and Classification System on Actinomycete Strains. Science Press, Beijing, 69-71.

[17] Wang, P. (1986) The Rapid Determination Method of Amino Acids and Simple Sugars on Actinomycete StrainsThin Layer Chromatography. Journal of Microbiology, 13, 228-230.

[18] Xu, L.-H., Li, W.-J., Liu, Z.-H., et al. (2007) The Principle, Method and Practice on System Science on Actinomycete Strains. Science Press, Beijing, 40-45.

[19] Xu, P., Li, W.-J. and Xu, L.-H. (2003) A Microwave-Based Method for Genomic DNA Extraction from Actinomycetes. Microbiology Reported, 3, 82-85.

[20] Ding, H.-G. (2006) The Causes and Prevention Measures on Bacterial Degradation and Aging. Special Economic Animals and Plants, 9, 39-40.

[21] Xi, J.-Q., Song, J.-Z., Zhou, H.-P., et al. (2006) Isolation and Screening of Chitinase Producing Strains and Inhibition Action to Pathogenic Fungi of the Same. Tobacco Science and Technology, 3, 55-58.

[22] Yue, H.-M., Yang, Q. and Song, J.-Z. (2009) Study on Analysis of Biological Control Effect of Two Kinds of Chaetomium spp. on Several Pathogen. Journal of Harbin University of Commerce (Natural Sciences Edition), 25, 593-596.

[23] Xu, P., Takahashi, Y.K., Seino, A., Iwai, Y., et al. (2004) Streptomyces scabrisporus sp. Nov. International Journal of Systematic and Evolutionary Microbiology, 54, 577-581. http://dx.doi.org/10.1099/ijs.0.02897-0 